\title{
Sm2, a paralog of the Trichoderma cerato-platanin elicitor Sm1, is also highly important for plant protection conferred by the fungal-root interaction of Trichoderma with maize
}

\author{
Romana Gaderer ${ }^{1 \dagger}$, Netta L Lamdan³, Alexa Frischmann', Michael Sulyok², Rudolf Krska², Benjamin A Horwitz ${ }^{3}$
} and Verena Seidl-Seiboth ${ }^{1 *}$

\begin{abstract}
Background: The proteins Sm1 and Sm2 from the biocontrol fungus Trichoderma virens belong to the cerato-platanin protein family. Members of this family are small, secreted proteins that are abundantly produced by filamentous fungi with all types of life-styles. Some species of the fungal genus Trichoderma are considered as biocontrol fungi because they are mycoparasites and are also able to directly interact with plants, thereby stimulating plant defense responses. It was previously shown that the cerato-platanin protein Sm1 from T. virens - and to a lesser extent its homologue Epl1 from Trichoderma atroviride - induce plant defense responses. The plant protection potential of other members of the cerato-platanin protein family in Trichoderma, however, has not yet been investigated.

Results: In order to analyze the function of the cerato-platanin protein $\mathrm{Sm} 2, \mathrm{sm} 1$ and $s m 2$ knockout strains were generated and characterized. The effect of the lack of Sm1 and Sm2 in T. virens on inducing systemic resistance in maize seedlings, challenged with the plant pathogen Cochliobolus heterostrophus, was tested. These plant experiments were also performed with $T$. atroviride ep/1 and ep/2 knockout strains. In our plant-pathogen system T. virens was a more effective plant protectant than T. atroviride and the results with both Trichoderma species showed concordantly that the level of plant protection was more strongly reduced in plants treated with the $\mathrm{sm} 2 / \mathrm{ep} / 2$ knockout strains than with sm1/ep/1 knockout strains.

Conclusions: Although the cerato-platanin genes sm1/ep/1 are more abundantly expressed than sm2/ep/2 during fungal growth, Sm2/Epl2 are, interestingly, more important than Sm1/Epl1 for the promotion of plant protection conferred by Trichoderma in the maize-C. heterostrophus pathosystem.
\end{abstract}

Keywords: Cerato-platanin protein, Trichoderma virens, Trichoderma atroviride, Mycoparasitism, Biocontrol, Plant protection, Maize, Cochliobolus heterostrophus

\section{Background}

Fungi belonging to the ascomycete genus Trichoderma inhabit the soil and rhizosphere, where they interact with plant roots and with other fungi. Agricultural biocontrol applications take advantage of the well-known ability of Trichoderma spp. to attack and destroy fungal

\footnotetext{
* Correspondence: verena.seidl@tuwien.ac.at

${ }^{+}$Equal contributors

'Research Division Biotechnology and Microbiology, Institute of Chemical Engineering, Vienna University of Technology, Gumpendorfer Strasse 1a, 1060 Vienna, Austria

Full list of author information is available at the end of the article
}

hosts, which is called mycoparasitism. The wide host range includes soil-borne plant pathogens such as Rhizoctonia solani or Pythium ultimum, which make Trichoderma spp. biological plant protectants. In addition, the interaction of Trichoderma with roots primes the plant's immune system for better resistance against pathogens [1-4]. Due to this induced systemic resistance, Trichoderma spp. are able to protect plants against some foliar pathogens, in addition to soil-borne pathogens. Plants recognize proteins secreted by the fungus, and such microbe associated (in this case fungal- 
associated) molecular patterns activate systemic resistance. The first such secreted protein to be studied in detail in Trichoderma-plant interactions was a small secreted cysteine-rich protein belonging to the cerato-platanin protein (CPP) family, named Sm1/Epl1 in Trichoderma virens and Trichoderma atroviride, respectively [5,6]. The Trichoderma genomes analyzed so far contain three genes encoding CPPs [7]. Gene expression was analyzed in $T$. atroviride and revealed that epl1 is expressed during hyphal growth, epl2 expression was only detected during spore maturation, and hardly any expression was found for epl3 [8]. Single and double knockout strains of epl1 and epl2 did not reveal any phenotype related to hyphal growth or development.

$T$. atroviride and $T$. virens belong to distant clades within the genus Trichoderma, for which so far already more than 200 species have been described [9]. Analysis of the genomes of $T$. virens and $T$. atroviride revealed numerous differences in the genome inventory of these two species, which each have more than 2500 genes that do not occur in the other species [7]. Further, even strongly conserved genes, e.g. chitinases, have already been shown to be differentially expressed in T. atroviride and $T$. virens [10]. However, the biological consequences of these findings on the lifestyle of $T$. atroviride and $T$. virens have, as yet, only been partially understood. It is therefore important to note that it is not always valid to draw direct conclusions from the results in one species to the other. Rather, one can study protein families in both of them in order to better elucidate the similarities and differences between $T$. atroviride and T. virens.

One example of the differences between these two species are the CPP orthologues Sm1 and Epl1. T. virens Sm1 was shown to induce plant defense responses, but this ability is far weaker for its homologue T. atroviride Epl1 [11]. This was explained by different tendencies of these proteins to dimerize. Only monomers of Sm1 and Epl1 were shown to efficiently induce plant defense responses. While Epl1 is readily able to form dimers, Sm1 has a single glycosylation site that is not present in Epl1 and is predominantly found in its monomeric form, which is more effective in the induction of plant defense responses [11].

The ability of CPP family members of Trichoderma from other phylogenetic branches to promote systemic resistance in plants has not been studied yet. In this study, we generated knockout strains of $T$. virens $s m 1$ and $s m 2$ and analyzed them for developmental phenotypes and for their ability to induce resistance of maize to the Southern corn leaf blight pathogen Cochliobolus heterostrophus. Plant experiments were also carried out with $T$. atroviride epl1 and epl2 knockout strains [8].

\section{Results}

Gene expression of CPP-encoding genes in $T$. virens

The genome of $T$. virens Gv29-8 contains three genes, $s m 1, s m 2$ and $s m 3$, encoding CPPs (http://genome.jgi-psf. org/TriviGv29_8_2/TriviGv29_8_2.home.html and [7]). This is in analogy to the genomes of T. atroviride and T. reesei, which also contain three CPP genes $[6,8]$. The genes $s m 2$ and $s m 3$ and their respective proteins have not been studied yet. In order to assess the transcriptional profiles of all three genes encoding CPPs in T. virens, their expression was first analyzed with RT-PCR. While the $s m 1$ gene was found to be expressed during hyphal growth, no expression was observed for $s m 2$ and $s m 3$ at these time points (Figure 1a). In biomass harvested from sporulating cultures grown on potato dextrose agar (PDA) plates during different stages of spore maturation, ranging from mycelium covered with white conidia to first light green and then dark green conidia, $s m 2$ was found to be strongly upregulated, but $s m 1$ was also found to be expressed (Figure 1b). For $s m 3$ no expression was detected under the tested growth conditions.

\section{Generation and characterization of $s m 1$ and $s m 2$ knockout strains}

Based on these gene expression results, gene knockout strains of $s m 1$ and $s m 2$ were generated in $T$. virens I10 $\Delta t k u 70$ [12] as described in the methods section (Additional file 1: Figure S1). Since Sm1 was previously shown to be far more potent in inducing plant defense responses than Epl1 [11] and the lack of $s m 1$ strongly reduces the ability of $T$. virens to induce plant defense responses [13], we were interested to test whether Sm2 has a similar function in T. virens. Phylogenetically Sm2 belongs to a different branch of CPPs [6] and none of these proteins have been studied so far. Since $s m 1$ is expressed throughout different growth stages and a possible function of CPPs in fungal growth has been discussed in the literature [14], the generated knockout strains were tested for phenotypic alterations with respect to the following properties related to fungal growth: growth on agar plates (Figure 2), formation of

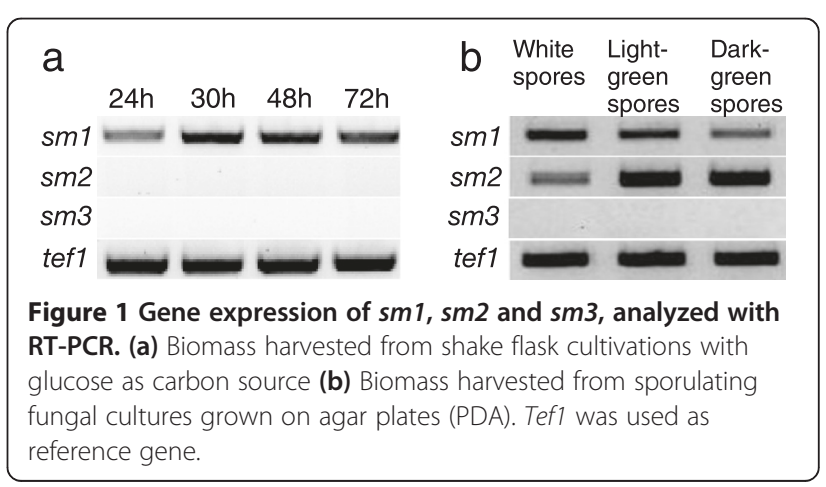




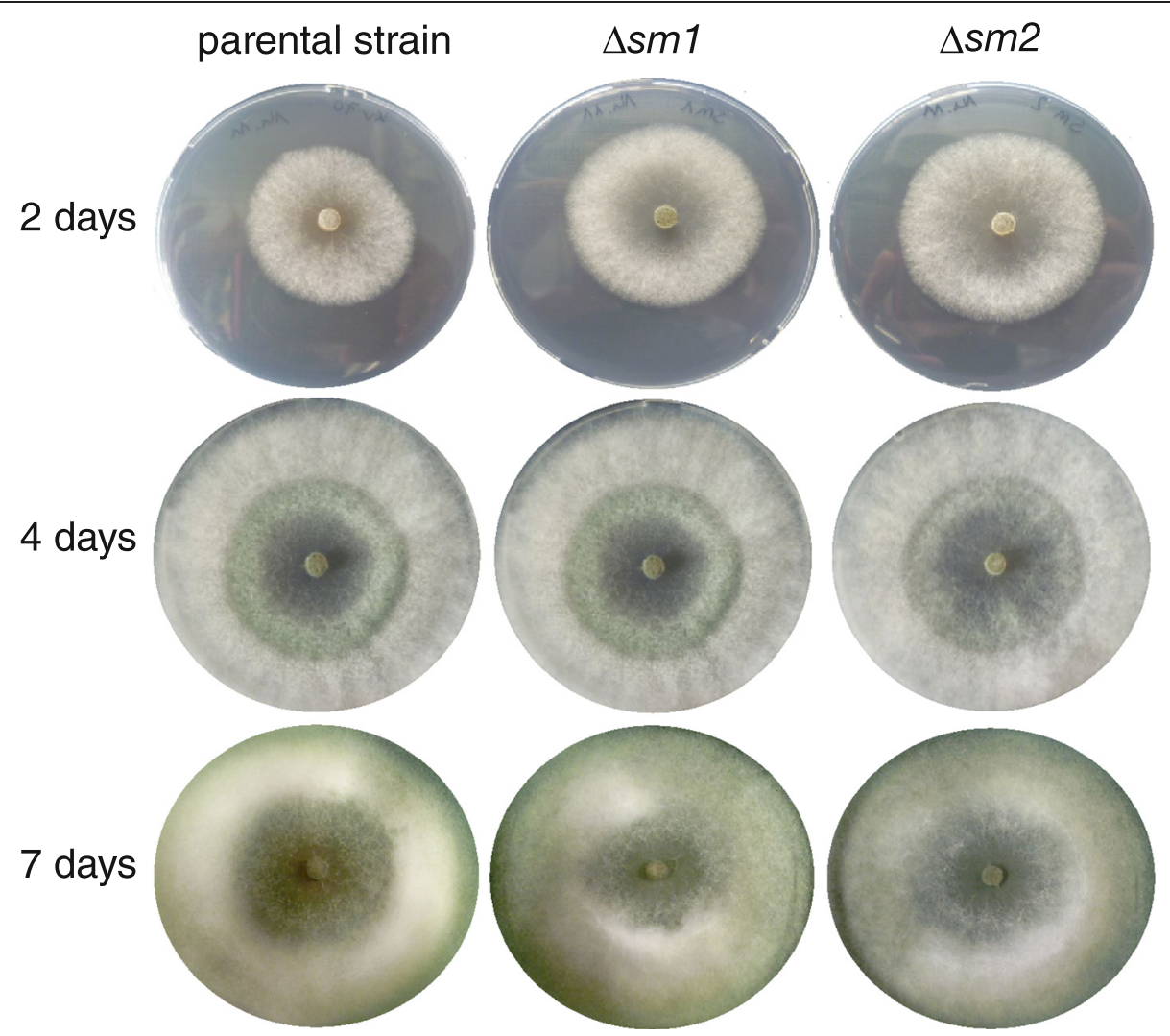

Figure 2 Colony growth of $T$. virens parental strain $\Delta t k u 70, s m 1$ and $s m 2$ knockout strains on agar plates (PDA).

aerial hyphae, growth along (moist) surfaces, bridging of gaps between two agar blocks, and transition of hyphae between solid/liquid interfaces. Different types of desiccation stress, e.g. drying of water droplets and drying of thin agar plates, were also examined. Furthermore, we analyzed conidiation, biomass formation in shake flask cultivations, germination efficiency, hydrophobicity of the mycelium, chlamydospore formation, osmotic stress, and cell wall stress (i.e. addition of Calcofluor white and Congo Red, both of which interfere with the construction and stress response of the cell wall [15]). No morphological differences between the parental strain and the knockout strains were found in T. virens, which is similar to $T$. atroviride, where no phenotype had been detected in epl1 and epl2 single and double knockout strains [8]. At advanced time points ( $48 \mathrm{~h}$ and $72 \mathrm{~h}$ ) of shake flask cultivations of $T$. virens abundant formation of chlamydospores was detected (Additional file 1: Figure S2). A correlation between chlamydospore formation and $c p$ (cerato-platanin) gene expression had been reported for Ceratocystis platani [16], but no differences were detected between the parental strain and the knockout strains in $T$. virens.

The mycoparasitic potential of the knockout strains was also not altered based on confrontation assays of $T$. virens against $R$. solani (Additional file 1: Figure S3) and Botrytis cinerea (data not shown).

We had found in a previous study that the expression of $T$. atroviride epl1, the homologue of $T$. virens sm1, is not constant during hyphal growth but its expression level is strongly dependent on parameters that influence the growth rate (e.g. medium composition and growth temperature) [8]. We therefore paid particular attention to this aspect in the knockout strains and assessed the gene expression of $s m 1$ quantitatively with qPCR in the $s m 2$ knockout strain and the parental strain $\Delta t k u 70$ (control strain). The results (Figure 3) showed that $\operatorname{sm} 1$ has a slightly different expression profile in the sm2 knockout strain compared to the parental strain, but we were not able to elucidate this further due to the lack of any detectable morphological changes in the knockout strains.

\section{$T$. virens strain $\mathbf{I} 10$ is a Q-strain}

$T$. virens strains can be grouped into P- and Q-strains, based on their antibiotic profiles. Strains of the Q-group produce the antibiotic gliotoxin and are generally considered to be more effective biocontrol agents [17]. The spectrum of secondary metabolites that is produced has a profound effect on the plant protection potential and 


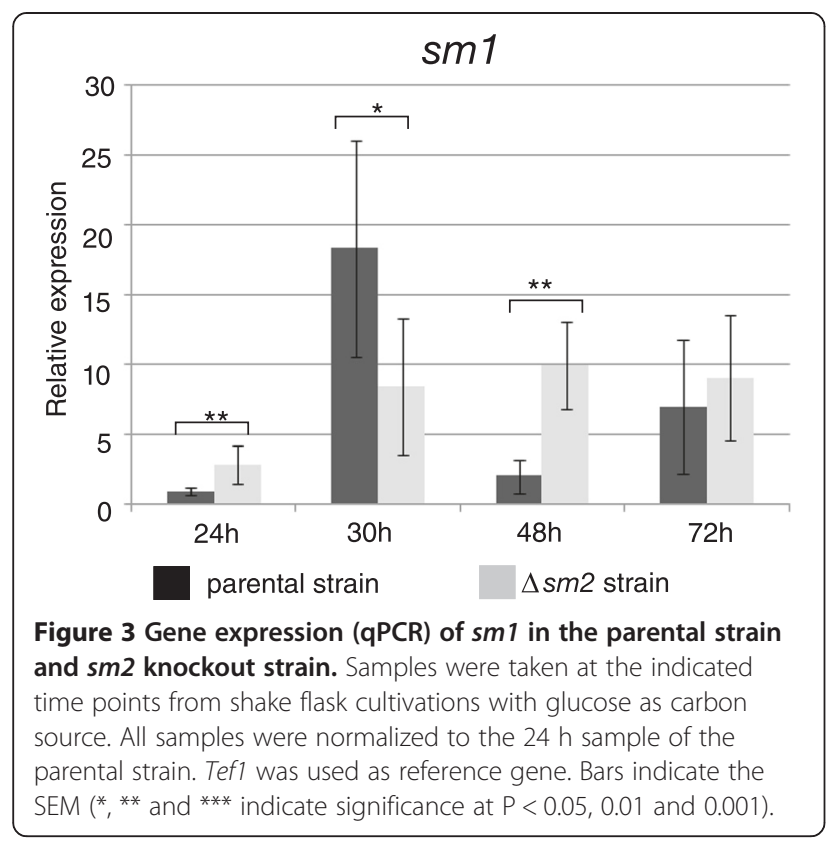

mycoparasitic activity of $T$. virens $[18,19]$. Previous studies on Sm1 were carried out in strain Gv29-8, which is a Q-strain [19]. In order to relate our experiments on the role of Sm2 (and Sm1) in the interaction of T. virens with plants (see below) more directly to previous studies $[5,13]$, we were interested whether strain I10, which we used for our studies [12], is a P or a Q-strain. T. virens sm1 - but not $s m 2$ - knockout strains have been previously studied in strain Gv29-8. Both strains, I10 and Gv29-8, were grown for $36 \mathrm{~h}$ in shake flask cultivations in liquid potato dextrose broth medium and gliotoxin production was measured from filtered culture supernatants. The results (Additional file 1: Figure S4) showed that I10 produced $18.5 \mathrm{mg} / \mathrm{l}$ gliotoxin, an amount similar to strain Gv29-8 (17.5 mg/l), and strain I10 can therefore also be attributed to the group of Q-strains of $T$. virens, which is of particular relevance for the discussion of our plant experiments (see below).

\section{Sm 1 and $s m 2$ knockout strains show reduced levels of plant protection}

In order to test whether $\mathrm{Sm} 2$ is involved in the interaction of Trichoderma with plants we analyzed whether the lack of $s m 2$ leads to an altered potential to protect plants against fungal pathogens. For this, the interaction of $C$. heterostrophus with its host, maize, was used as a model pathosystem. We have recently standardized this system (N.L.L. and B.A.H., unpublished results) and it was used previously with Trichoderma asperellum ([20]). The $T$. virens parental and $\operatorname{sm} 1$ knockout strains were also included, and in addition experiments were performed with $T$. atroviride wild-type, epl1 and epl2 knockout strains generated in a previous study [8]. Lesion sizes on maize plants, whose roots were co-cultured with the $T$. virens parental strain $\Delta t k u 70$ or $s m 1$ and $s m 2$ knockout strains, were measured (Figure 4a, b). Colonization of maize roots by the $T$. virens parental strain significantly decreased symptoms by more than $40 \%$. Plants colonized by $\Delta s m 1$ showed impaired resistance in comparison to plants treated with the $T$. virens parental strain, but were still significantly different from control plants, showing about 30\% decrease in lesion size. Knock-out of $s m 2$, however, led to a much more dramatic decrease in the ability of the fungus to induce resistance in maize, resulting in large lesions which were similar to the lesions of the control plants (no Trichoderma). In order to assess the gene expression of sm-genes in T. virens during plant interaction, root biomass was harvested from plant experiments four days post inoculation with $T$. virens conidia and analyzed by qPCR. Loss of one sm-gene might affect the expression of the other through feedback in the signaling network. The results (Figure 4c) showed, overall, no strong changes in gene expression of the other sm-gene. The expression of $s m 1$ was not altered in the $\Delta s m 2$ strain. $s m 2$ expression tended to be higher in $\Delta s m 1$ than in the parental strain, but this was not statistically significant.

Plant experiments were also carried out with $T$. atroviride epl1 and epl2 single and double knockout strains that were generated in a previous study [8]. While plants treated with the T. virens parental strain showed more than $40 \%$ reduction in lesion size, the lesion size in plants treated with the T. atroviride IMI206040 wild-type strain was only $13 \%$ smaller than in the control plants (Figure 4d). Despite the relatively poor plant protection potential of $T$. atrovir$i d e$, we observed exactly the same trend regarding ability of $\Delta e p l 1$ and $\Delta e p l 2$ strains to protect plants as for the corresponding mutants in $T$. virens. In addition, lesion sizes on plants colonized by the double knockout strain $\Delta e p l 1 \Delta e p l 2$ were not significantly different from $\Delta e p l 2$ treated plants and control plants (Figure 4d).

\section{Discussion}

In this study the gene expression of the three genes encoding CPPs in $T$. virens was analyzed. In analogy to previous results from $T$. atroviride, $\operatorname{sm} 1$ was found to be expressed during hyphal growth, i.e. under growth conditions when sufficient nutrients are available and fast biomass formation occurs. For $s m 2$ gene expression was detected in mycelium that was harvested from sporulating cultures. Since the formation of conidia is associated with differentiation of the hyphae to form conidiophores and phialides, sm2 gene expression could also be associated with these structures, but it is unfortunately not possible to separate them efficiently. It should be noted that in shake flask cultivations, where, according to our microscopic observations, large amounts of chlamydospores - which directly split off from hyphae - but no 

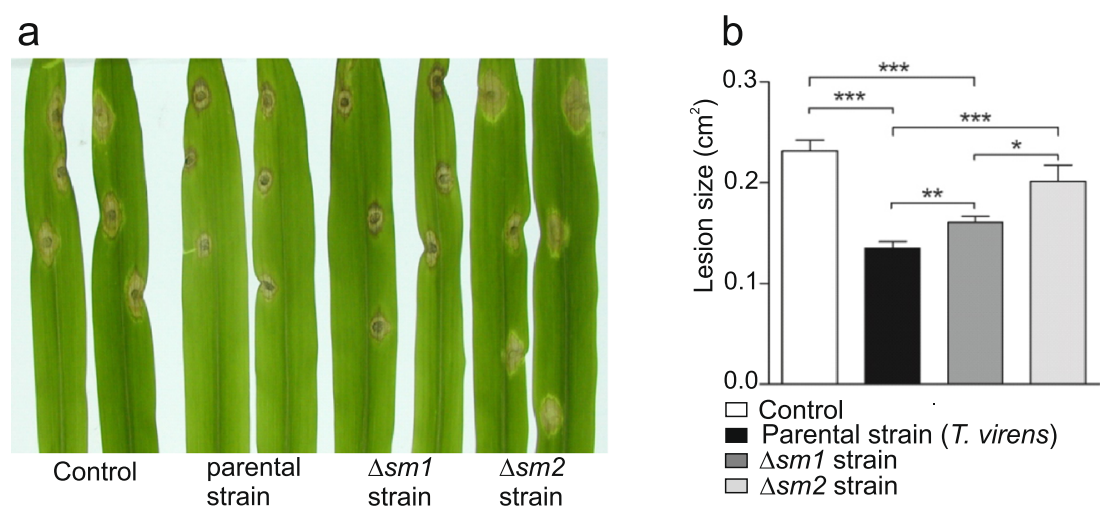

C

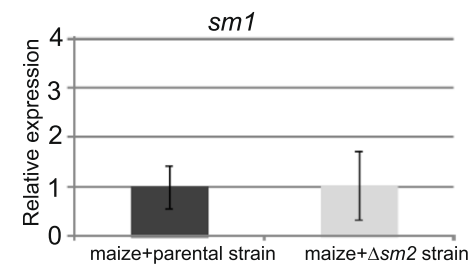

d

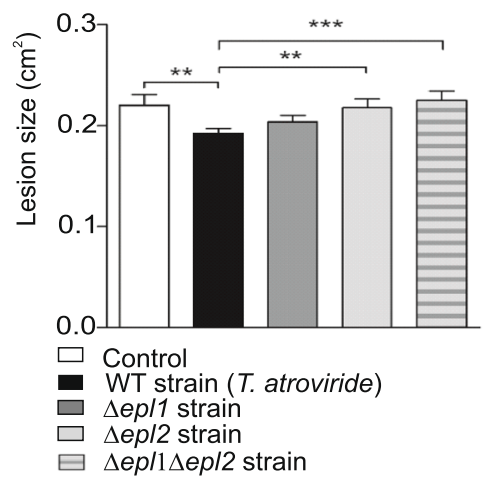

Figure 4 Effect of $T$. virens (parental strain, $\Delta s m 1$ and $\Delta s m 2$ ) on plant protection in maize seedlings challenged with the maize pathogen C. heterostrophus. (a) Lesion development in leaves of $T$. virens-induced maize, two days after pathogen challenge. (b) Lesion size measured from photographed leaves. Number of lesions measured in control plant, 33; T. virens parental strain, 23; $\Delta s m 1,24 ; \Delta s m 2$, 23. (c) Gene expression (qPCR) of $s m 1$ and $s m 2$ in maize seedlings infected with the pathogen C. heterostrophus and treated with the T. virens parental strain, sm1 and sm2 knockout strain. Number of lesions measured in control plant, 24; T. atroviride wild-type, 27; $\Delta$ ep/1, 23; $\Delta$ ep/2, $23 ; \Delta e p / 1 \Delta e p / 2,24$. Data from (b) and (d) represent the combined data from plants taken from two beakers. The experiment was carried out independently twice, with two biological repeats, each one containing 12 plants, with the same results within the variability. Bars indicate the SEM (standard error of the mean). ${ }^{*}, * *$ and ${ }^{* * *}$ indicate significance at $\mathrm{P}<0.05,0.01$ and 0.001. (d) Effect of T. atroviride (wild-type, $\Delta e p / 1, \Delta e p / 2$, and $\left.\Delta e p / 1 \Delta e p / 2\right)$ on plant protection in maize seedlings challenged with the maize pathogen C. heterostrophus. Lesion size measured from photographed leaves.

conidia, were formed, no expression of $\operatorname{sm} 2$ was found (Figure 1 and Figure S2 in supplemental material). This indicates that $s m 2$ gene expression is connected to the formation and maturation of conidia but not to other types of spores in $T$. virens. It should be noted that, due to morphological differences of the mycelium on agar plates between $T$. atroviride and $T$. virens, in $T$. atroviride the harvested biomass from sporulating cultures consists mainly of spores, whereas in T. virens I10 the mycelium is fluffier and the harvested biomass is therefore a mixture of spores and hyphae. This probably explains why in $T$. virens expression of $s m 1$ and $s m 2$ was detected in these samples, whereas in T. atroviride strong epl2 expression but only weak epl1 expression was found. Sm2 is also expressed in co-culture with maize (Figure 4).

Our transcriptional data for $\operatorname{sm} 1$ are in agreement with findings by Djonovic et al. [5], who reported expression under all tested conditions, including sporulating and non-sporulating mycelia. In other fungi there is also evidence that homologues of $s m 1$ are expressed during hyphal growth, e.g. in B. cinerea, bcspll was found to be expressed under many different growth conditions, whereas no expression was found for $b c s p l 2$, a second CP gene [21]. MgSM1 from Magnaporthe grisea was also expressed during different fungal growth stages [22]. In addition to these gene expression data, the protein Epl1 was found to be the predominant protein in the secretome of submerged $T$. atroviride cultivations with glucose as a carbon source [6]. In the plant pathogenic basidiomycete Moniliophthora perniciosa gene expression data of the $12 \mathrm{CP}$ genes (MpCP1-12) showed complex transcriptional profiles throughout fungal development and pathogenic infestation of the plant, suggesting a specialization of the respective proteins in different biological processes 
[23]. In ascomycetes, gene expression data are so far limited to homologues of $s m 1$ except for epl 2 and epl3 from T. atroviride and $b c s p l 2$ from $B$. cinerea (see above). It will be of interest for future studies to obtain more expression data for these genes, in particular considering the strong effect of the lack of $s m 2$ on the ability of Trichoderma to protect maize from $C$. heterostrophus in our plant experiments (Figure 4).

The mycoparasitic potential of $s m$-knockout strains against $R$. solani and $B$. cinerea was not altered and it can be anticipated that this would also be the case for other host fungi. Nonetheless $s m$-knockout strains have a strong effect on the biocontrol properties of $T$. virens via direct effects on the Trichoderma-plant interaction. In the T. virens Gv29.8-maize - Colletotrichum graminicola interaction, loss of Sm1 resulted in complete loss of the capacity to reduce lesion size on the leaves [13]. A single protein might seem unlikely to be responsible, alone, for the induction of systemic resistance. However, it seems that in the maize C. graminicola interaction $\mathrm{Sm} 1$ is indeed the dominant player. In the maize Cochliobolus assay used in this study, on the other hand, knock-out of $\operatorname{sm} 1$ reduced the plant protection potential of $T$. virens, but in this pathosystem the lack of Sm2 had an even greater effect and lesion size of maize leaves was statistically not different from the control (no Trichoderma). Although the colonization efficiency of maize roots by $T$. virens was not directly measured, it should be noted that upon harvesting of plant roots for biomass extraction for qPCR experiments, no obvious phenotypes in fungal growth and the appearance of the colonized roots were observed. In plant experiments with $T$. atroviride plant protection levels were overall lower than with $T$. virens, but the same trend was observed for epl1 and epl2 knockout strains as for $\operatorname{sm} 1$ and $\sin 2$ knockout strains, confirming that $\mathrm{Sm} 2 / \mathrm{Epl} 2$ are, in the C. heterostrophus-maize pathosystem, more important for plant protection than $\mathrm{Sm} 1 / \mathrm{Ep} 1$. This is also relevant because it underlines that the observed effect is not due to any unwanted, genetic side-effects of the knockout strains or due a particular feature of the $T$. virens strain used. The $T$. atroviride double knockout strain $\Delta e p l 1 \Delta e p l 2$ appeared slightly (albeit not significantly) less effective than the $\Delta e p l 2$ strain (Figure $4 \mathrm{~b}, \mathrm{~d}$ ). These data are compatible with an additive contribution of Sm1 and Sm2, and the contribution of these two paralogs to the induction of resistance is similar for $T$. virens and $T$. atroviride. The data in Figure 4 provide genetic evidence that for maximal induction of resistance, both paralogs need to be present. In contrast to maize- C. graminicola, where $\mathrm{Sm} 1$ dominates, Sm2 appears to be the dominant one in this particular assay. Since $s m 1$ expression was not found to be altered in the sm2 deletion strain in plant experiments it can be concluded that reduction of the capacity to protect the plant was directly due to the absence of Sm2.

When comparing the C. graminicola to the C. heterostrophus pathosystem assays, it is important to note that the assay here was done using hydroponic cultures rather than soil-grown plants, which could affect the relative extent of colonization, intensity of ISR and contribution of specific secreted proteins. To maximize the potential of Trichoderma spp. to protect plants, expression of different combinations of CPP family members, at different levels, will need to be tested, in different pathosystems.

\section{Conclusions}

CPPs are potent inducers of plant defense responses in plant pathogenic fungi as well as plant-beneficial fungi such as Trichoderma species. In this study we showed that $T$. virens $s m 2$ knockout strains were more impaired in the protection of maize seedlings against the pathogen C. heterostrophus than $s m 1$ knockout strains. T. atroviride was overall less effective in plant protection than $T$. virens, but the same trend was observed for the respective epl2 and epl1 knockout strains. These findings advance our understanding of the diversified functions of CPPs in fungi and of the pool of molecules that are involved in the beneficial interaction of Trichoderma with plants. Our results show that the paradigm of Sm1 as the main or exclusive inducer of plant systemic resistance triggered by Trichoderma-root interactions needs to be generalized. As we have shown that in the particular interaction studied here $\mathrm{Sm} 2$ is even more important than $\mathrm{Sm} 1$, it seems likely that even more elicitors remain to be discovered in Trichoderma.

\section{Methods}

\section{Generation of knockout strains and phenotype analysis}

Knockout strains were generated using T. virens I10 $\Delta t k u 70[12]$ as a parental strain. A schematic representation of the $\operatorname{sm} 1$ and $\operatorname{sm} 2$ knockout loci is shown in Additional file 1: Figure S1. All primers used for generation and verification of knockout strains are listed (see Additional file 1: Table S1). For construction of the $\operatorname{sm1}$ deletion vector $900 \mathrm{bp}$ of the 5'- and $860 \mathrm{bp}$ of the 3'flanking regions of $\operatorname{sm} 1$ were amplified from genomic DNA of $T$. virens 110 with the primers sm1-5'-fw/sm15'-rv and sm1-3'-fw/sm1-3'-rv, respectively. The PCR products were cloned into a pBS (Bluescript $\mathrm{SK}+$ ) vector containing a selection hph-cassette ( $h p h$ gene, conferring resistance to hygromycin, under control of the $T$. reesei pki promoter and cbh2 terminator) [24]. The obtained plasmid was first linearized with XhoI and the sm15 flanking region was inserted with the In-Fusion cloning kit (Clontech, Mountain View, CA, USA). Then the resulting plasmid was again linearized with EcoRV and the sm1-3' flanking region was inserted. For identification 
of knockout strains primers sm1-promupstream-fw and sm1-hph-cass-rv were used, yielding a $2 \mathrm{~kb}$ PCR product for positive knockout strains. The purification of the knockout strains and thus absence of the $s m 1$ gene was verified with primers sm1-promupstream-fw and sm1term-rv, yielding a PCR product with the size of $2 \mathrm{~kb}$ for the sm1-wild-type.

For generation of the sm2 deletion vector the Aspergillus oryzae ptrA gene was used as a selection marker, conferring resistance against pyrithiamine [25]. The resistance marker cassette, containing the native promoter and terminator of the ptr gene, was amplified from a plasmid (kindly obtained from B. Seiboth) and inserted into a pBS (Bluescript $\mathrm{SK}+$ ) vector that was previously linearized with XhoI and HindIII via In-Fusion cloning (Clontech). The 5 -flanking region of $s m 2$ was amplified with the primers sm2-5'-fw/sm1-5'-rv and the 3'-flanking region was amplified with the primers sm2-3'-fw/sm2-3'-rv. The flanking regions were inserted into plasmid pBS-ptr that was linearized with XhoI for the sm2-5' region and HindIII for the sm2-3' region via In-Fusion cloning (Clontech). Knockout strains were identified by PCR with the primers sm2promupstream-fw and sm2-ptr-cass-rv, yielding a $2 \mathrm{~kb}$ band for the $s m 2$ knockout locus. After single spore isolations, the absence of the $s m 2$ gene was verified with the primers sm2-promupstream-fw and sm2-term-rv, yielding a $2.2 \mathrm{~kb}$ band for the $s m 2$ wild-type locus.

Fungal transformation, carried out with the PCR-amplified transformation cassettes, protoplast generation, preparation of selection media and purification of fungal transformants were performed as described in [12]. For selection on pyrithiamine $(1 \mu \mathrm{g} / \mathrm{ml}) T$. virens transformants were grown on ISM medium; $0.68 \mathrm{~g} / \mathrm{L} \mathrm{KH}_{2} \mathrm{PO}_{4}$, $0.87 \mathrm{~g} / \mathrm{L} \mathrm{K} \mathrm{KPO}_{4}, 1.7 \mathrm{~g} / \mathrm{L}\left(\mathrm{NH}_{4}\right)_{2} \mathrm{SO}_{4}, 0.2 \mathrm{~g} / \mathrm{L} \mathrm{CaCl}_{2}$, $0.2 \mathrm{~g} / \mathrm{L} \mathrm{KCl}, 0.2 \mathrm{~g} / \mathrm{L} \mathrm{MgSO}_{4} \cdot 7 \mathrm{H}_{2} \mathrm{O}, 5 \mathrm{mg} / \mathrm{L} \mathrm{FeSO}_{4} \cdot 7 \mathrm{H}_{2} \mathrm{O}$, $2 \mathrm{mg} / \mathrm{L} \mathrm{ZnSO}_{4} \cdot 7 \mathrm{H}_{2} \mathrm{O}, 2 \mathrm{mg} / \mathrm{L} \mathrm{MnSO}_{4} \cdot 7 \mathrm{H}_{2} \mathrm{O}$ [6] in order to facilitate the differentiation between transformants and background growth.

Mycoparasitism assays were performed on potato dextrose agar (PDA) plates. T. virens and a host fungus ( $R$. solani or B. cinerea) were placed on opposite sides of the agar plate and incubated at $28^{\circ} \mathrm{C}$ with a $12 \mathrm{~h} / 12 \mathrm{~h}$ light/dark cycle. Images of the confrontation assays were taken every $24 \mathrm{~h}$ to record the antagonism and overgrowth of the host fungi by $T$. virens.

Phenotype analysis of the knockout strains was carried out as described for T. atroviride in [8]. All experiments were carried out with at least two independent biological replicates.

\section{Fungal cultivations and gene expression analysis}

Shake flask cultivations were carried out with ISM medium [6] containing $1 \%$ glucose and $0.05 \%$ peptone. Media were inoculated with $1 \times 10^{6}$ conidia $/ \mathrm{ml}$ and cultivated at $25^{\circ} \mathrm{C}$ and $200 \mathrm{rpm}$. Mycelia were harvested at the time points indicated in the results section and frozen in liquid nitrogen. For gene expression analysis from conidia at different maturation stages (based on the appearance of the mycelium covered with conidia, ranging from white to first light green and then dark green conidia), sporulated mycelia were scraped from PDA plates with a spatula and frozen in liquid nitrogen. For RNA isolation the samples were ground to a fine powder under liquid nitrogen and total RNA was isolated using the guanidinium thiocyanate method [26]. Isolated RNAs were treated with DNAse I (Fermentas, St Leon-Rot, Germany), and cDNAs were generated with the Revert Aid $\mathrm{H}$-minus cDNA synthesis kit (Fermentas). RT-PCR ( 25 cycles) was performed using the gene-specific primers listed (see Additional file 1: Table S1). Accession numbers of the sm-genes in the JGI T. virens genome database; http://genome.jgi-psf.org/ TriviGv29_8_2/TriviGv29_8_2.home.html) are: $\operatorname{sm} 1$ 110852, sm2 111830 and sm3 32154. The corresponding accession numbers in the NCBI database are EHK25601 for Sm1, EHK20677 for Sm2, and EHK25819 for Sm3. The tef1 gene (translation elongation factor 1 alpha, protein ID 83874 in the JGI database and in the NCBI database EHK22702) was used as reference gene.

qPCR reactions were performed in an Eppendorf Realplex thermal cycler. The reaction mix contained $12.5 \mu$ S SYBR green Supermix (Bio-Rad Laboratories, Hercules, CA, USA), $8.5 \mu \mathrm{l}$ pure water, $6.25 \mu \mathrm{M}$ forward and $6.25 \mu \mathrm{M}$ reverse primer, and $2 \mu \mathrm{l}$ 1:50 diluted template cDNA ( $5 \mu \mathrm{g}$ of RNA/reaction were reversetranscribed using the Revert Aid H-minus cDNA synthesis kit (Fermentas)). Reactions were performed in triplicates. Primer efficiency was calculated using a dilution series from 1:5 to 1:5000 with the PCR baselinesubtracted mode. The amplification protocol consisted of an initial denaturation step for $3 \mathrm{~min}$ at $95^{\circ} \mathrm{C}$ followed by 40 cycles of denaturation $\left(95^{\circ} \mathrm{C}\right.$ for $\left.15 \mathrm{~s}\right)$, annealing, and elongation $\left(60^{\circ} \mathrm{C}\right.$ for $\left.15 \mathrm{~s}\right)$. Oligonucleotides are listed in Additional file 1: Table S1. The tef1 gene was used as a reference. Expression data were evaluated using REST software [27]. Cultivations for gene expression analysis were carried out with at least two independent biological replicates.

\section{Gliotoxin measurements}

LC-MS/MS screening of fungal metabolites was performed with a QTrap 5500 LC-MS/MS System (Applied Biosystems, Foster City, CA) equipped with a TurbolonSpray electrospray ionization (ESI) source and a 1290 Series HPLC System (Agilent, Waldbronn, Germany). Chromatographic separation was performed at $25^{\circ} \mathrm{C}$ on a $\mathrm{Gemini}^{\circ} \mathrm{C}_{18^{-}}$ column, $150 \times 4.6 \mathrm{~mm}$ i.d., $5 \mu \mathrm{m}$ particle size, equipped with a $\mathrm{C}_{18} 4 \times 3 \mathrm{~mm}$ i.d. security guard cartridge (all from Phenomenex, Torrance, CA, US). The chromatographic 
method, as well as chromatographic and mass spectrometric parameters for 186 of the investigated analytes, is as described by Vishwanath et al. [28]. In the meantime, the method has been further expanded to cover 320 metabolites.

ESI-MS/MS was performed in the time-scheduled multiple reaction monitoring (MRM) mode both in positive and negative polarities in two separate chromatographic runs per sample by scanning two fragmentation reactions per analyte. The MRM detection window of each analyte was set to its expected retention time \pm 27 seconds and \pm 48 seconds in the positive and the negative mode, respectively. The target cycle time was 1 second. Confirmation of positive analyte identification was obtained by the acquisition of two MRMs per analyte (with the exception of moniliformin and 3-nitropropionic acid, that exhibit only one fragment ion), which yielded 4.0 identification points according to commission decision 2002/657/EC. In addition, the LC retention time and the intensity ratio of the two MRM transition agreed with the related values of an authentic standard within $0.1 \mathrm{~min}$ and $30 \%$ rel., respectively.

For further confirmation of the identity of gliotoxin, Enhanced Product Ion scans were performed using the third quadrupole as linear ion trap, applying a collision energy of $35 \mathrm{~V}$, a collision energy spread of $15 \mathrm{~V}$ and a dynamic fill time of the trap. Spectra were obtained by averaging 20 scans of a scan speed of $1000 \mathrm{amu} / \mathrm{sec}$ and a scan range of $50-820 \mathrm{amu}$.

\section{Plant assays for induced systemic resistance}

We used a hydroponic system $[5,29]$ to evaluate the resistance response of maize seedlings stimulated by $T$. virens or $T$. atroviride. $600 \mathrm{ml}$ glass beakers were filled with $200 \mathrm{ml}$ plant nutrient solution (half-strength Murashige and Skoog basal medium, $2.5 \mathrm{mM}$ MES buffer $\mathrm{pH}=5.7$ ). A perforated stand for supporting the seeds was made from a $200 \mu \mathrm{l}$ tip holder. Maize seeds (Royalty, local hybrid, purchased from Ben Shachar, Tel Aviv) were surface sterilized by dipping them in $10 \% \mathrm{H}_{2} \mathrm{O}_{2}$ for three hours, followed by three washes with sterile water. Treated seeds were dried on sterile Whatman \#1 paper, placed in sterile Petri dishes containing half-strength Murashige and Skoog agar and incubated in the dark for three days at $30^{\circ} \mathrm{C}$ to allow germination. 12 germinated seeds with similar-sized roots and shoots were placed on the stands in each aseptic beaker. The plants were maintained in a controlled environment at $23^{\circ} \mathrm{C}$ and a $16 \mathrm{~h}$ photoperiod with moderate shaking on an orbital shaker (100 rpm). After four days of growth in the beakers, plants were inoculated with Trichoderma spore suspension to a final concentration of $5 \times 10^{3}$ spores $/ \mathrm{ml}$. Roots and fungus were allowed to interact for four more days before pathogen challenge. For pathogen challenge, plants - with their roots - were taken out of the beakers and the second leaf of each plant was attached to a tray from the edges of the leaf. The roots of each set of plants according to treatment were wrapped separately in wet paper towels.

The maize pathogen $C$. heterostrophus (strain C4) was grown for seven days on complete xylose medium [30] in the same controlled environment as the plants. The second leaf was inoculated with $7 \mu \mathrm{l}$ droplets of $0.02 \%$ Tween 20 in double distilled water containing 1000 spores. Trays were closed in clear plastic bags to keep the plants moist and kept in the controlled environment. Pictures of the challenged leaves were taken after $48 \mathrm{~h}$ and lesions were measured using ImageJ software (http://imagej.nih.gov/ij/). For each treatment the data represent at least eight leaves, each with three lesions from two biological repeats.

For analysis of gene expression, roots with adhering Trichoderma were harvested from hydroponic cultures grown in parallel to those used for the ISR assays. The roots were washed gently with culture medium, frozen and ground to a fine powder in liquid nitrogen. RNA was extracted using Tri Reagent (MBC Molecular Research Center, Cincinnati, OH, USA) following the manufacturer's protocol, and cDNA was synthesized as described above.

\section{Supporting data}

The data sets supporting the results of this article are included within the article and its additional files.

\section{Additional file}

Additional file 1: Figure S1. Generation of $s m 1$ and $s m 2$ knockout strains. Figure S2. Chlamydospore formation in T. virens shake flask cultivations. Figure S3. Mycoparasitism confrontation assays of T. virens against $R$. solani. Figure S4. Gliotoxin measurements of $T$. virens strains 110 and Gv29-8. Table S1. Primers used in this study.

\section{Abbreviations}

CPP: Cerato platanin protein; CP: Cerato platanin; PDA: Potato dextrose agar.

\section{Competing interests}

The authors declare that they have no competing interests.

\section{Authors' contributions}

RG carried out the gene expression analysis, generation and morphological characterization of knockout strains, NLL performed the plant experiments, AF contributed to the generation of knockout strains, MS and RK carried out the gliotoxin measurements, $\mathrm{BAH}$ participated in the design and writing of the manuscript, VSS designed the study, coordinated the experiments and writing of the manuscript. All authors read and approved the final manuscript.

\section{Acknowledgements}

This work was supported by grants T390 and P26028 of the FWF Austrian Science Fund to V.S-S. N.L.L. predoctoral fellowship from Technion graduate school, work in B.A.H. lab supported by the Technion Vice-President for Research (VPR) fund.

\section{Author details}

'Research Division Biotechnology and Microbiology, Institute of Chemical Engineering, Vienna University of Technology, Gumpendorfer Strasse 1a, 1060 Vienna, Austria. ${ }^{2}$ Department for Agrobiotechnology (IFA-Tulln), University of Natural Resources and Life Sciences (BOKU), Vienna, Austria. ${ }^{3}$ Department of Biology, Technion - Israel Institute of Technology, Haifa, Israel. 
Received: 26 September 2014 Accepted: 18 December 2014 Published online: 16 January 2015

\section{References}

1. Druzhinina IS, Seidl-Seiboth V, Herrera-Estrella A, Horwitz BA, Kenerley CM, Monte E, et al. Trichoderma: the genomics of opportunistic success. Nat Rev Microbiol. 2011;9:749-59.

2. Harman GE, Howell CR, Viterbo A, Chet I, Lorito M. Trichoderma species opportunistic, avirulent plant symbionts. Nat Rev Microbiol. 2004;2:43-56

3. Lorito M, Woo SL, Harman GE, Monte E. Translational research on Trichoderma: from 'omics to the field. Annu Rev Phytopathol. 2010;48:395-417.

4. Mukherjee PK, Horwitz BA, Herrera-Estrella A, Schmoll M, Kenerley CM. Trichoderma research in the genome era. Annu Rev Phytopathol. 2013;51:105-29.

5. Djonovic S, Pozo MJ, Dangott LJ, Howell CR, Kenerley CM. Sm1, a proteinaceous elicitor secreted by the biocontrol fungus Trichoderma virens induces plant defense responses and systemic resistance. Mol Plant Microbe Interact. 2006;19:838-53.

6. Seidl V, Marchetti M, Schandl R, Allmaier G, Kubicek CP. Epl1, the major secreted protein of Hypocrea atroviridis on glucose, is a member of a strongly conserved protein family comprising plant defense response elicitors. FEBS J. 2006;273:4346-59.

7. Kubicek CP, Herrera-Estrella A, Seidl-Seiboth V, Martinez DA, Druzhinina IS, Thon $\mathrm{M}$, et al. Comparative genome sequence analysis underscores mycoparasitism as the ancestral life style of Trichoderma. Genome Biol. 2011;12:R40.

8. Frischmann A, Neudl S, Gaderer R, Bonazza K, Zach S, Gruber S, et al. Self-assembly at air/water interfaces and carbohydrate binding properties of the small secreted protein EPL1 from the fungus Trichoderma atroviride. J Biol Chem. 2013;288:4278-87.

9. Atanasova L, Druzhinina IS, Jaklitsch WM. Two hundred Trichoderma species recognized based on molecular phylogeny. In: Mukherjee PK, Sigh US, Horwitz BA, Schmoll M, Mukherjee M, editors. Trichoderma: Biology and Applications. Wallingford, UK: CABl; 2013. p. 10-42.

10. Gruber S, Kubicek CP, Seidl-Seiboth V. Differential regulation of orthologous chitinase genes in mycoparasitic Trichoderma species. Appl Environ Microbiol. 2011;77:7217-26

11. Vargas WA, Djonovic S, Sukno SA, Kenerley CM. Dimerization controls the activity of fungal elicitors that trigger systemic resistance in plants. J Biol Chem. 2008;283:19804-15.

12. Catalano V, Vergara M, Hauzenberger JR, Seiboth B, Sarrocco S, Vannacci G, et al. Use of a non-homologous end-joining-deficient strain (delta-ku70) of the biocontrol fungus Trichoderma virens to investigate the function of the laccase gene Icc1 in sclerotia degradation. Curr Genet. 2011;57:13-23.

13. Djonovic S, Vargas WA, Kolomiets MV, Horndeski M, Wiest A, Kenerley CM. A proteinaceous elicitor Sm1 from the beneficial fungus Trichoderma virens is required for induced systemic resistance in maize. Plant Physiol. 2007; 145:875-89.

14. Gaderer R, Bonazza K, Seidl-Seiboth V. Cerato-platanins: a fungal protein family with intriguing properties and application potential. Appl Microbiol Biotechnol. 2014:98:4795-803.

15. Ram AF, Klis FM. Identification of fungal cell wall mutants using susceptibility assays based on Calcofluor white and Congo red. Nat Protoc 2006;1:2253-6.

16. Baccelli I, Comparini C, Bettini PP, Martellini F, Ruocco M, Pazzagli L, et al. The expression of the cerato-platanin gene is related to hyphal growth and chlamydospores formation in Ceratocystis platani. FEMS Microbiol Lett. 2012;327:155-63.

17. Howell CR. Selective isolation from soil and separation in vitro of $P$ and $Q$ strains of Trichoderma virens with differential media. Mycologia. 1999;91:930-4

18. Mukherjee PK, Horwitz BA, Kenerley CM. Secondary metabolism in Trichoderma-a genomic perspective. Microbiology. 2012;158:35-45

19. Vargas WA, Mukherjee PK, Laughlin D, Wiest A, Moran-Diez ME, Kenerley CM. Role of gliotoxin in the symbiotic and pathogenic interactions of Trichoderma virens. Microbiology. 2014;160:2319-30.

20. Enhancement of plant disease resistance by the biocontrol agent Trichoderma [http://www.weizmann.ac.il/Biology/open_day_2006/book/ Abstracts/llan_Chet.pdf]
21. Frías M, González C, Brito N. BcSpl1, a cerato-platanin family protein, contributes to Botrytis cinerea virulence and elicits the hypersensitive response in the host. New Phytol. 2011;192:483-95.

22. Yang $Y$, Zhang $H$, Li G, Li W, Wang $X$, Song F. Ectopic expression of MgSM1, a Cerato-platanin family protein from Magnaporthe grisea, confers broad-spectrum disease resistance in Arabidopsis. Plant Biotechnol J. 2009;7:763-77.

23. de O Barsottini MR, de Oliveira JF, Adamoski D, Teixeira PJ, do Prado PF, Tiezzi HO, et al. Functional diversification of cerato-platanins in Moniliophthora perniciosa as seen by differential expression and protein function specialization. Mol Plant Microbe Interact. 2013;26:1281-93.

24. Mach RL, Schindler M, Kubicek CP. Transformation of Trichoderma reesei based on hygromycin B resistance using homologous expression signals. Curr Genet. 1994;25:567-750.

25. Kubodera T, Yamashita N, Nishimura A. Transformation of Aspergillus sp. and Trichoderma reesei using the pyrithiamine resistance gene (ptrA) of Aspergillus oryzae. Biosci Biotechnol Biochem. 2002;66:404-6.

26. Sambrook J, Russell DW. Molecular Cloning: a Laboratory Manual. 2nd ed. Painview, NY: Cold Spring Harbor Lab. Press; 2001.

27. Pfaffl MW, Horgan GW, Dempfle L. Relative expression software tool (REST) for group-wise comparison and statistical analysis of relative expression results in real-time PCR. Nucleic Acids Res. 2002;30:e36.

28. Vishwanath V, Sulyok M, Labuda R, Bicker W, Krska R. Simultaneous determination of 186 fungal and bacterial metabolites in indoor matrices by liquid chromatography/tandem mass spectrometry. Anal Bioanal Chem. 2009:395:1355-72.

29. Yedidia II, Benhamou N, Chet II. Induction of defense responses in cucumber plants (Cucumis sativus L.) by the biocontrol agent Trichoderma harzianum. Appl Environ Microbiol. 1999;65:1061-70.

30. Turgeon BG, Condon B, Liu J, Zhang N. Protoplast transformation of filamentous fungi. In: Sharon A, editor. Methods Mol Biol. New York: Humana/Springer; 2010. p. 3-19 [Walker J (Series Editor): Springer Protocols: Methods in Molecular Biology].

\section{Submit your next manuscript to BioMed Central and take full advantage of:}

- Convenient online submission

- Thorough peer review

- No space constraints or color figure charges

- Immediate publication on acceptance

- Inclusion in PubMed, CAS, Scopus and Google Scholar

- Research which is freely available for redistribution 